Pacific Journal of Mathematic 


\title{
PERTURBATION OF THE CONTINUOUS SPECTRUM AND UNITARY EQUIVALENCE
}

\author{
MARVIN RosenBLUm
}

1. Introduction. Suppose that $A$ and $B$ are self-adjoint operators in a Hilbert space $H$ such that $B-A=P$ is a completely continuous operator. We shall concern ourselves with the problem of finding conditions sufficient to guarantee that $B$ is unitarily equivalent to $A$. Clearly a necessary condition is that the spectrum of $A$ (considered as a point set on the real line) is equal to the spectrum of $B$. However this condition is not sufficient; von Neumann [8] has proved the following result

1.1. Let $A$ and $C$ be bounded self-adjoint operators in a separable Hilbert space, such that the spectra of $A$ and $C$ have the same limit points. Then there exists an operator $B$ that is unitarily equivalent to $C$ and such that $B-A$ is completely continuous.

Thus we see that perturbation by a completely continuous operator can radically alter the multiplicity of the spectrum. Even if $A$ and $B$ have pure continuous spectra on the same interval, it does not follow that $B$ is unitarily equivalent to $A$.

Our present investigation continues along lines begun by Friedrichs in [1] and [2]. He considered bounded operators $A$ that have continuous spectrum of finite multiplicity, and worked in the representation space where $A$ corresponds to a multiplication operator. One of Friedrichs' results is the following.

1.2. Let $H=L^{2}(-1,1)$ and let $A$ be the operator that sends any function $f(x)$ of $H$ into $x f(x)$.. Let $P$ be the integral operator with the hermitian kernel $p(x, y)=\overline{p(y, x)}$, where $p$ satisfies certain Lipschitz conditions. Then if $\varepsilon$ is a sufficiently small real number, there exist unitary operators $U_{\varepsilon}$ and $V_{\varepsilon}$ such that

(i) $e^{-i(A+\varepsilon P) t} e^{i A t}$ converges strongly to $U_{\varepsilon}$

as $t \rightarrow \infty$;

(ii) $e^{-i(A+\varepsilon P) t} e^{i A l}$ converges strongly to $V_{\varepsilon}$ as $t \rightarrow-\infty$;

Received April 23, 1956. Research performed in part under contract DA-04-200-ORD171 Task Order 5 for Office of Ordnance Research, U.S. Army. This paper is a revised version of the author's doctoral dissertation submitted to the University of California, Berkeley. He wishes to thank Professor Frantisek Wolf for his advice and encouragement. 
and

(iii) $U_{\varepsilon}{ }^{*}(A+\varepsilon P) U_{\varepsilon}=A$ and $V_{\varepsilon}{ }^{*}(A+\varepsilon P) V_{\varepsilon}=A$.

The operator $S=U_{\varepsilon}^{*} V_{\varepsilon}$ is the scattering operator, which is of intersect in quantum mechanics; see H. E. Moses [5] and Kay and Moses [4].

We shall make the following assumptions.

Assumption 1.3. $A=\int_{-\infty}^{\infty} x d_{x} E_{x}$ and $B=\int_{-\infty}^{\infty} x d_{x} F_{x}$ are (possibly unbounded) self-adjoint operators and $B-A=P$ is a completely continuous operator such that the trace of $|P|$ is finite'.

Assumption 1.4. The spectral measure of $A$ is weakly absolutely continuous, that is, $\left(E_{x} f, g\right)$ is an absolutely continuous function of $x$ for all $f, g$ in $H$.

We want to find conditions on $B$ that will guarantee that $B$ is unitarily equivalent to $A$, that is, that there exist a unitary operator $U$ such that $B U=U A$, or equivalently, that $\left(F_{x} U f, g\right)=\left(E_{x} f, U^{*} g\right)$ for all $f, g$ in $H$. Thus a necessary condition is given in

Assumption 1.5. The spectral measure of $B$ is weakly absolutely continuous.

We shall show in this paper that this condition is also sufficient. In fact, we shall prove the following.

Theorem 1.6. Suppose that 1.3, 1.4, and 1.5 hold. Then as $t \rightarrow \infty$, or $t \rightarrow-\infty, e^{-i B t} e^{i A t}$ converges strongly to unitary operators $U$ and $V$ respectively, such that $U^{*} B U=A$ and $V^{*} B V=A$.

By von Neumann's theorem (see 1.1) Theorem 1.6 is no longer true if $P$ is allowed to be an arbitrary completely continuous operator. It should be noticed that we have imposed no smallness condition on the norm of $P$, and that $A$ may have continuous spectrum of any multiplicity.

1. Sketch of the proof. Actually, to prove Theorem 1.6, we have only to prove the following (seemingly) weaker result.

2.1. Assume 1.3-1.5 hold. Then as $t \rightarrow \infty, e^{-i B t} e^{i \Delta t}$ converges weak-

1 For any self-adjoint $A=\int_{-\infty}^{\infty} \lambda d E_{\lambda}$ we define $|A|=\int_{-\infty}^{\infty}|\lambda| d E_{\lambda}$. 
ly to an operator $U$ such that $B U=U A$ and $\|U f\|=\|f\|$ for all $f$ in $H$.

Proof. We shall deduce Theorem 1.6 from 2.1. We assume that the hypotheses and conclusions of 2.1 are valid. Recall that if a sequence $\left\{g_{n}\right\}$ of elements of $H$ converges weakly to a limit element $g$, and if $\left\|g_{n}\right\| \rightarrow\|g\|$, then $g_{n}$ converges strongly to $g$.

Let $f \in H . \quad U_{\iota}=e^{-i B l} e^{i A l}$ is unitary, so

$$
\lim _{t \rightarrow \infty}\left\|U_{t} f\right\|=\|f\|=\|U f\| \text {. }
$$

But, since $U_{t} f$ also converges weakly to $U f$, it follows from the preceding paragraph that as $t \rightarrow \infty, U_{t} f$ converges strongly to $U f$. Also $f\|=\| U f \|$ implies that

$$
(f, f)=\|f\|^{2}=\|U f\|^{2}=(U f, U f)=\left(U^{*} U f, f\right), \quad \text { so } \quad U^{*} U=I .
$$

Now, 2.1 holds for all choices of $A$ and $B$ that satisfy the Assumptions 1.3-1.5. Since $B-A=P$ we see that $A-B=-P$. Thus we can substitute $A$ for $B, B$ for $A$, and $-P$ for $P$, and we can infer from 2.1 and the preceding paragraph that $e^{-i A t} e^{i B t}$ converges strongly to some operator $W$, and $W^{*} W=I$. Since $\left(e^{-i B t} e^{i A t}\right)^{*}=e^{-i A t} e^{i B t}$ we deduce that $W=U^{*}$ and that $U U^{*}=U^{*} U=I$, so that $U$ is unitary and $U^{*} B U$ $=A$.

It is also true that $-B-(-A)=-P$, and if we substitute $-A$ for $A$ and $-B$ for $B$ in 2.1 we can repeat the above arguments to prove that as $t \rightarrow \infty, e^{i B t} e^{-i A t}$ converges strongly to a unitary operator $V$ such that $V^{*} B V=A$.

In the remainder of this paper we prove 2.1. From now on we assume that assumptions $1.3-1.5$ hold. We know that $P=B-A$ has a representation

$$
P=\sum_{j=1}^{\infty} \lambda_{j}\left(\cdot, \phi_{j}\right) \phi_{j},
$$

where the $\phi_{j}$ are orthonormal and

$$
\sum_{j=1}^{\infty}\left|\lambda_{j}\right|<\infty
$$

We put $U_{t}=e^{-i B t} e^{i A t}$. For any complex-valued Lebesgue-measurable function $K(x)$ that is almost everywhere finite we can define the normal operator $K(A)$ by specifying that

$$
(K(A) f, g)=\int_{-\infty}^{\infty} K(x)^{d\left(E_{x} f, g\right)} d x
$$

for suitable $f$ in $H$. In particular, $\left(e^{-i \Delta t} f, g\right)$ is the Fourier transform 
of $d\left(E_{x} f, g\right) / d x$. The letters $f, g$ shall denote arbitrary elements in $H$. The following sketch of our method of attack may prove instructive. We first derive the representation theorem

3.3. (i) $\left(U_{t} f, g\right)=(f, g)+\frac{1}{i} \int_{0}^{t}\left(e^{-i B x} P e^{i A x} f, g\right) d x$.

We wish to take $t \rightarrow \infty$ and thus exhibit an operator $U$ such that $U_{t}$ converges weakly to $U$. But the integrand in 3.3 (i) is not necessarily integrable over $(0, \infty)$. However, we show in 4.4 (i) and 4.7 that there exists a function $w(x)$ that is finite a. e. and such that when $f$ is in the domain of $w(A)$ and $g$ is in the domain of $u(B)$, then the integrand in 3.3 (i) belongs to $L(0, \infty)$. Using this fact we prove in part 5 that $U_{t}$ converges to an operator $U$ such that $B U=U A$.

Now we have to show that $\|U f\|=\|f\|$. We proceed in an indirect fashion. Rather than work with $U_{t}$ we consider the operators $K_{n}(B) U_{t}$, where the $K_{n}(x)$ are a sequence of characteristic functions such that $K_{n}(x) \rightarrow 1$ and such that the integrand in the representation

$$
\left(K_{n}(B) U_{t} f, g\right)=\left(K_{n}(B) f, g\right)+\frac{1}{i} \int_{0}^{t}\left(K_{n}(B) e^{-i B x} P e^{i A x} f, g\right) d x
$$

belongs to $L(0, \infty)$ for a dense set of $f$ and all $g$ in $H$. We show that, for each $n, K_{n}(B) U_{t}$ converges strongly to $K_{n}(B) U$, and thus

$$
\lim _{n \rightarrow \infty} \lim _{t \rightarrow \infty}\left\|K_{n}(B) U_{t} f\right\|=\|U f\| \text {. }
$$

By means of representation Theorem 3.5 we show that this iterated limit is also equal to $\|f\|$, and thus $\|U f\|=\|f\|$, and 2.1 is proved.

\section{Derivation of the representation theorems.}

LEMMA 3.1. If $s$ is a complex number with nonzero real part, then

$$
\begin{aligned}
(s+i B)^{-1} & =(s+i A)^{-1}-i(s+i B)^{-1} P(s+i A)^{-1} \\
& =(s+i A)^{-1}-i(s+i A)^{-1} P(s+i B)^{-1}
\end{aligned}
$$

Proof. Since $B-A$ is bounded it follows that $A$ and $B$ have the same domain $D$. Then, for any $f \in H,(s+i B)^{-1} f \in D$, and

$$
\begin{aligned}
-i(s+i A)^{-1} P(s+i B)^{-1} f & =(s+i A)^{-1}(s+i A-s-i B)(s+i B)^{-1} f \\
& =(s+i B)^{-1} f-(s+i A)^{-1} f .
\end{aligned}
$$

(ii) follows similarly. 
LEMMA 3.2. If $s$ is a complex number with nonzero real part, and if $L$ is a bounded operator that commutes with $(s+i B)^{-1}$, then

(i) $(s+i A)^{-1} L-L(s+i A)^{-1}=-i(s+i A)^{-1}[L P-P L](s+i A)^{-1}$.

Proof. By 3.1 (i),

$$
\begin{aligned}
L(s+i B)^{-1} & =L(s+i A)^{-1}-i L(s+i B)^{-1} P(s+i A)^{-1} \\
& =L(s+i A)^{-1}-i(s+i B)^{-1} L P(s+i A)^{-1} .
\end{aligned}
$$

By 3.1 (ii), this last expression equals

$$
L(s+i A)^{-1}-i(s+i A)^{-1} L P(s+i A)^{-1}-(s+i A)^{-1} P(s+i B)^{-1} L P(s+i A)^{-1} .
$$

It can be similarly shown that

$$
\begin{aligned}
(s+i B)^{-1} L= & (s+i A)^{-1} L-i(s+i A)^{-1} P L(s+i A)^{-1} \\
& -(s+i A)^{-1} P L(s+i B)^{-1} P(s+i A)^{-1} .
\end{aligned}
$$

Lemma 3.2 follows upon subtracting this last equation from the preceding equation and using the commutativity property of $L$.

In the following representation theorems all operator integrals are understood to be defined in the weak sense.

Theorem 3.3. For any real number $t$,

(i) $U_{t}=I+\frac{1}{i} \int_{0}^{t} e^{-i B x} P e^{i A x} d x$, and

(ii) $e^{-i B t}=e^{-i A t}+\frac{1}{i} \int_{0}^{t} e^{-i B x} P e^{i A(x-t)} d x$.

Proof. Let $s$ be a complex number with positive real part. Then 3.1 (i) holds and

$$
\int_{0}^{\infty} e^{-s t} e^{-i A t} d t=(s+i A)^{-1}
$$

for any self-adjoint operator $A$. Hence by the Laplace transform, convolution and uniqueness theorems as found in Hille [3], chapter 10, we derive (ii).

(i) follows from (ii) by operating on the right with $e^{i A t}$.

THEOREM 3.4. If $L$ is a bounded operator that commutes with every bounded function of $B$, then

(i) $e^{-i A t} L e^{i A t}=L+\frac{1}{i} \int_{0}^{t} e^{-i A x}(L P-P L) e^{i A x} d x$. 
Proof. We start with 3.2, parallel the proof of Theorem 3.3, and derive the formula

$$
e^{-i A t} L-L e^{-i A t}=\frac{1}{i} \int_{0}^{t} e^{-i A x}(L P-P L) e^{i A(x-t)} d x .
$$

3.4 follows by operating on the right with $e^{i A t}$.

We shall use 3.4 (i) in the following form.

COROLLARY 3.5. If $K$ is a projection operator that commutes with every bounded function of $B$, then

(i) $\left\|K U_{t} f\right\|^{2}-\|f\|^{2}=([K-I] f, f)+\frac{1}{i} \int_{0}^{t}\left(e^{-i A x}(K-I) P e^{i A x} f, f\right) d x$

$$
-\frac{1}{i} \int_{0}^{t}\left(e^{-i A x} P(K-I) e^{i A x} f, f\right) d x
$$

Proof. We set $L=K-I$ in 3.4 (i) and take inner products. Then the right hand side of 3.4 (i) is equal to the right hand side of 3.5 (i). But,

$$
\begin{aligned}
\left(e^{-i A t}[K-I] e^{i A t} f, f\right) & =\left(e^{-i A t} K e^{i A t} f, f\right)-(f, f) \\
& =\left\|K e^{i A t} f\right\|^{2}-\|f\|^{2} \\
& =\left\|K U_{t} f\right\|^{2}-\|f\|^{2},
\end{aligned}
$$

so the left sides are equal which proves 3.5.

4. Definition of the $K_{n}(x)$.

THEOREM 4.1.

(i ) $0 \leqq \frac{d\left(E_{x} f, f\right)}{d x}<\infty$ for almost all $x$;

(ii) $\int_{-\infty}^{\infty}\left|\frac{d\left(E_{x} f, g\right)}{d x}\right| d x \leqq\|f\| \cdot\|g\|$

(iii) $\int_{-\infty}^{\infty} d\left(E_{x} f, g\right) d x=(f, g)$; and

(iv) $\left|\frac{d\left(E_{x} f, g\right)}{d x}\right|^{2} \leqq \frac{d\left(E_{x} f, f\right)}{d x} \cdot \frac{d\left(E_{x} g, g\right)}{d x}$ for almost all $x$.

Proof. (i) follows from the fact that $\left(E_{x} f, f\right)$ is a monotone increasing function of $x$; 
(ii) is true because the total variation of $\left(E_{x} f, g\right)$ is $\leq\|f\| \cdot\|g\|$, (see Riesz and Nagy [6, p. 340]).

(iii) holds because $E_{\infty}=I$ and $E_{-\infty}=0$.

We shall now derive (iv). If $h$ is a nonzero real number, then

$$
\left|\left(\left[E_{x+h}-E_{x}\right] f, g\right)\right|^{2}=\frac{1}{h^{2}}\left|\left(\left[E_{x+h}-E_{x}\right] f,\left\lceil E_{x+h}-E_{x}\right] g\right)\right|^{2},
$$

which by the Schwarz inequality is

$$
\leqq\left(\left[\frac{E_{x+h}-E_{x}}{h}\right] f, f\right) \cdot\left(\left[\frac{E_{x+h}-E_{x}}{h}\right] g, g\right) .
$$

Taking $h$ to 0 completes the proof.

LEMMA 4.2.

(i) $\int_{-\infty}^{\infty}\left|\left(e^{i A x} f, g\right)\right|^{2} d x \leqq 2 \pi \int_{-\infty}^{\infty}\left|\frac{d\left(E_{x} f, g\right)}{d x}\right|^{2} d x$

(ii) $\lim _{t \rightarrow \infty}\left(e^{i A t} f, g\right)=0$.

Proof. If $d\left(E_{x} f, g\right) / d x$ is not square integrable, then the right hand side is infinite and there is nothing to prove. If $d\left(E_{x} f, g\right) / d x$ is square integrable, then its Fourier transform is also square integrable and (i) is an equality.

(ii) is a consequence of 4.1 (ii) and the Riemann-Lebesgue lemma.

LEMmA 4.3. If $Q$ is a bounded self-adjoint operator, then

(i ) $\int_{-\infty}^{\infty}\left\||P|^{\frac{1}{2}} Q e^{i A x} f\right\|^{2} d x \leqq 2 \pi \int_{-\infty}^{\infty}\left[\sum_{j=1}^{\infty}\left|\lambda_{j}\right| d\left(E_{x} Q \phi, Q \phi_{j}\right) / d x\right] d\left(E_{x} f, f\right) / d x d x$ $\leqq 2 \pi \operatorname{ess} \sup d\left(E_{x} f, f\right) / d x \sum_{j=1}^{\infty}\left|\lambda_{j}\right|\left\|Q \phi_{j}\right\|^{2}$

(ii) $\int_{-\infty}^{\infty}\left\||P|^{\frac{1}{2}} Q e^{i B x} f\right\|^{2} d x \leqq 2 \pi \int_{-\infty}^{\infty}\left[\sum_{j=1}^{\infty}\left|\lambda_{j}\right| d\left(F_{x} Q \phi_{j}, Q \phi_{j}\right) / d x\right] d\left(F_{x} f, f\right) / d x d x$

Proof. In this proof we have nonnegative integrands and thus we freely commute integration and summation.

$$
\int_{-\infty}^{\infty}\left\||P|^{\frac{1}{2}} Q e^{i A x} f\right\|^{2} d x=\int_{-\infty}^{\infty} \sum_{j=1}^{\infty}\left|\lambda_{j}\right|\left|\left(e^{i A x} f, Q \phi_{j}\right)\right|^{2} d x,
$$

which by 4.2 (i) and then 4.1 (iv) is

$$
\leqq 2 \pi \int_{-\infty}^{\infty} \sum_{j=1}^{\infty}\left|\lambda_{j}\right|\left|d\left(E_{x} f, Q \phi_{j}\right) / d x\right|^{2} d x
$$




$$
\leq 2 \pi \int_{-\infty}^{\infty}\left[\sum_{j=1}^{\infty}\left|\lambda_{j}\right| d\left(E_{x} Q \phi_{j}, Q \phi_{j}\right) / d x\right] d\left(E_{x} f, f\right) / d x d x
$$

The remaining inequality in (i) is readily derived from this and 4.1 (iii).
4.3.
(ii) follows similarly.

LEMMA 4.4. If $Q_{1}$ and $Q_{2}$ are bounded self-adjoint operators and $-\infty \leqq s, t \leqq \infty$, then

(i) $\left[\int_{s}^{t}\left|\left(e^{-i B x} P e^{i A x} f, g\right)\right| d x\right]^{2} \leqq \int_{s}^{t}\left\||P|^{\frac{1}{2}} e^{i A x} f\right\|^{2} d x \int_{-\infty}^{\infty}\left\||P|^{\frac{1}{2}} e^{i B x} g\right\|^{2} d x$.

(ii) $\left[\int_{-\infty}^{\infty}\left|\left(e^{-i A x} Q_{1} P Q_{2} e^{i A x} f, f\right)\right| d x\right]^{2}$

$$
\begin{aligned}
\leqq\left[2 \pi \operatorname{ess} \sup d\left(E_{x} f, f\right) / d x\right]\left[\sum_{j=1}^{\infty}\left|\lambda_{j}\right|\right. & \left.\left\|Q_{1} \phi_{j}\right\|^{2}\right] \\
& \times\left[\sum_{j=1}^{\infty}\left|\lambda_{j}\right|\left\|Q_{2} \phi_{j}\right\|^{2}\right] .
\end{aligned}
$$

Proof. Let $C$ be the operator $A$ or the operator $B$. Since for any self-adjoint operator $P$ we have the decomposition $P=|P|^{\frac{1}{2}}$ sgn $P|P|^{\frac{1}{2}}$, we have

$$
\begin{aligned}
\left|\left(e^{-i C x} Q_{1} P Q_{2} e^{i A x} f, g\right)\right| & =\left|\left(e^{-i C x} Q_{1}|P|^{\frac{1}{2}} \operatorname{sgn} P|P|^{\frac{1}{2}} Q_{2} e^{i A x} f, g\right)\right| \\
& =\left|\left(\operatorname{sgn} P|P|^{\frac{1}{2}} Q_{2} e^{i A x} f,|P|^{\frac{1}{2}} Q_{1} e^{i \sigma x} g\right)\right|,
\end{aligned}
$$

which by the Schwarz inequality and the fact that $\|\operatorname{sgn} P\| \leq 1$ is

$$
\leqq\left\||P|^{\frac{1}{2}} Q_{2} e^{i A x} f\right\| \cdot\left\||P|^{\frac{1}{2}} Q_{1} e^{i c x} g\right\|
$$

By the Schwarz inequality for integrals and the above calculation we see that

$$
\begin{aligned}
{\left[\int_{s}^{t}\left|\left(e^{-i \delta x} Q_{1} P Q_{2} e^{i A x} f, g\right)\right| d x\right]^{2} } \\
\leq \int_{s}^{t}\left\||P|^{\frac{1}{2}} Q_{2} e^{i \Delta x} f\right\|^{2} d x \int_{s}^{t}\left\||P|^{\frac{1}{2}} Q_{1} e^{i \sigma x} g\right\|^{2} d x \\
\leq \int_{s}^{t}\left\||P|^{\frac{1}{2}} Q_{2} e^{i \Delta x} f\right\|^{2} d x \int_{-\infty}^{\infty}\left\||P|^{\frac{1}{2}} Q_{1} e^{i C x} g\right\|^{2} d x .
\end{aligned}
$$

If we put $Q_{1}=Q_{2}=I$ and $C=B$ we see that we have derived (i). If we put $t=\infty, s=-\infty, C=A$, and employ 4.3 (i), we derive (ii). 
DEFINITION 4.5.

$$
w(x)=\sum_{j=1}^{\infty}\left|\lambda_{j}\right|\left[d\left(E_{x} \phi_{j}, \phi_{j}\right) / d x+d\left(F_{x} \phi_{j}, \phi_{j}\right) / d x\right] .
$$

THEOREM 4.6. For almost all $x, w(x)$ is nonnegative and finite.

Proof. From 4.1 (i) and the definition of $w(x)$ it follows that $w(x)$ is nonnegative a.e.

$$
\int_{-\infty}^{\infty} w(x) d x=\sum_{j=1}^{\infty}\left|\lambda_{j}\right|\left[\int_{-\infty}^{\infty} d\left(E_{x} \phi_{j}, \phi_{j}\right) / d x d x+\int_{-\infty}^{\infty} d\left(F_{x} \phi_{j}, \phi_{j}\right) / d x d x\right]
$$

which by 4.1 (iii), is $2 \sum_{j=1}^{\infty}\left|\lambda_{j}\right|$. This last term is finite by assumption. Hence $w(x)$ is integrable, and thus is a. e. finite.

LemMa 4.7. If $f$ is in the domain of $w(A)$, then

(i) $\int_{-\infty}^{\infty}\left\||P|^{\frac{1}{2}} e^{i A x} f\right\|^{2} d x \leqq 2 \pi(w(A) f, f)$.

If $f$ is in the domain of $w(B)$, then

(ii) $\int_{-\infty}^{\infty}\left\||P|^{\frac{1}{2}} e^{i B x} f\right\|^{2} d x \leqq 2 \pi(w(B) f, f)$.

Proof. By 4.3 (i)

$$
\begin{aligned}
& \int_{-\infty}^{\infty}\left\||P|^{\frac{1}{2}} e^{i \Delta x} f\right\|^{2} d x \\
& \quad \leq 2 \pi \int_{-\infty}^{\infty}\left[\sum_{j=1}^{\infty}\left|\lambda_{j}\right| d\left(E_{x} \phi_{j}, \phi_{j}\right) / d x\right] d\left(E_{x} f, f\right) / d x d x,
\end{aligned}
$$

which is

$$
\leqq 2 \pi \int_{-\infty}^{\infty} w(x) d\left(E_{x} f, f\right) / d x d x=2 \pi(w(A) f, f) .
$$

(ii) follows similarly.

Definition 4.8. For every positive integer $n$, let $K_{n}(x)$ be the characteristic function of the set of real number $x$ such that $w(x)=\infty$ or $w(x) \leqq n$.

TheOREM 4.9. For every positive integer $n$,

(i) $K_{n}(x)$ is a measurable function

(ii) $\left[K_{n}(x)\right]^{2}=K_{n}(x)$ and $K_{n}(x)$ is real; 
(iii) $\lim _{n \rightarrow \infty} K_{n}(x)=1$; and

(iv) $0 \leqq w(x) K_{n}(x) \leqq n$ for almost all $x$.

Proof. (i)-(iii) follow immediately from the definition of $K_{n}(x)$. (iv) is a consequence of 4.6 .

THEOREM 4.10. For every positive integer $n$,

(i ) $K_{n}(A)$ is a projection operator such that

(ii) $0 \leqq w(A) K_{n}(A) \leqq n$;

(iii) $\lim _{n \rightarrow \infty}\left(K_{n}(A) f, g\right)=(f, g)$; and

(iv) $\lim _{n \rightarrow \infty}\left\|K_{n}(A) f-f\right\|=0$.

(i)-(iv) also hold when $A$ is replaced everywhere by $B$.

Proof. (i)-(iii) are direct consequences of 4.9. (iv) follows from (iii).

THEOREM 4.11. Let $n$ be any positive integer. Then as $t \rightarrow \infty$, $K_{n}(B) U_{t}$ converges strongly.

Proof. For $f$ in the domain of $w(A)$ and all $g$ in $H$

$$
\left|\left(K_{n}(B)\left[U_{t}-U_{s}\right] f, g\right)\right|^{2}=\left|\left(\left[U_{t}-U_{s}\right] f, K_{n}(B) g\right)\right|^{2},
$$

which by 3.3 (i) and then 4.4 (i) and 4.7 (ii)

$$
\begin{aligned}
= & \left|\frac{1}{i} \int_{s}^{t}\left(e^{-i B x} P e^{i A x} f, K_{n}(B) g\right) d x\right|^{2} \\
& \leqq 2 \pi \int_{s}^{t}\left\||P|^{\frac{1}{2}} e^{i A x} f\right\|^{2} d x \cdot\left(w(B) K_{n}(B) g, K_{n}(B) g\right) .
\end{aligned}
$$

But by 4.10 (ii) this is

$$
\leqq 2 \pi \int_{s}^{t}\left\||P|^{\frac{1}{2}} e^{i A x} f\right\|^{2} d x \cdot n \cdot\|g\|^{2} .
$$

Now set $g=K_{n}(B)\left[U_{t}-U_{s}\right] f$ in preceding inequality. When then have

$$
\begin{aligned}
& \left\|K_{n}(B)\left[U_{t}-U_{s}\right] f\right\|^{4} \\
& \quad \leq 2 \pi \int_{s}^{t}\left\||P|^{\frac{1}{2}} e^{i \Delta x} f\right\|^{2} d x \cdot n \cdot\left\|K_{n}(B)\left[U_{t}-U_{s}\right] f\right\|^{2} \\
& \quad \leq 8 \pi n\|f\|^{2} \int_{s}^{t}\left\||P|^{\frac{1}{2}} e^{i A x} f\right\|^{2} d x
\end{aligned}
$$


But, by 4.7 (i) the integrand in this last expression belongs to $L(-\infty$, $\infty)$. Thus $\lim _{s, t \rightarrow \infty}\left\|K_{n}(B)\left[U_{t}-U_{s}\right] f\right\|=0$.

We have proved that for all $f$ in a dense set, $K_{n}(B) U_{t} f$ converges strongly. Since $\left\|K_{n}(B) U_{t}\right\| \leq 1$, it follows that 4.11 is true.

\section{The Operator $U$.}

THEOREM 5.1. As $t \rightarrow \infty, U_{t}$ converges weakly to an operator $U$. For any $f, g$ in $H$,

(i) $(U f, g)=(f, g)-i \lim _{t \rightarrow \infty} \int_{0}^{t}\left(e^{-i B x} P e^{i \Delta x} f, g\right) d x$.

Proof. We know from 3.3 (i) that

$$
\left(U_{t} f, g\right)=(f, g)-i \int_{0}^{t}\left(e^{-i B x} P e^{i A x} f, g\right) d x .
$$

The estimates 4.4 (i) and 4.7 assure us that the integrand in this expression belongs to $L(0, \infty)$ for all $f$ in the domain $D_{1}$ of $w(A)$ and all $g$ in the domain $D_{2}$ of $w(B)$. Thus the bilinear form

$$
b(f, g)=b(f, g)=\lim _{t \rightarrow \infty}\left(U_{t} f, g\right)
$$

is defined on $D_{1} \times D_{2}$. Since $\left\|U_{t}\right\|=1$ this form is bounded and it follows from the Frechet-Riesz representation theorem (see Stone [7], p. 63) that there exists a bounded, everywhere defined operator $U$ such that $(U f, g)=b(f, g)$ for all $f, g$ in $D_{1} \times D_{2}$. In fact, since the $U_{t}$ are uniformly bounded it is the case that $(U f, g)=\lim _{t \rightarrow \infty}\left(U_{t} f, g\right)$ for all $f, g$ in H. Thus 5.1 (i) holds.

LEMMA 5.2. For all $f$ in $H, \lim _{t \rightarrow \infty}\left\|P e^{i A t} f\right\|=0$.

Proof. Let $\varepsilon>0$. Since $P$ is completely continuous there exists an integer $n$ and an operator

$$
P_{n}=\sum_{j=1}^{n} \lambda_{j}\left(\cdot, \phi_{j}\right) \phi_{j}
$$

such that $\left\|P-P_{n}\right\| \leqq \varepsilon$. Then

$$
\begin{gathered}
\left\|P e^{i A t} f\right\|=\left\|\left(P_{n}+P-P_{n}\right) e^{i A t} f\right\| \leqq\left\|P_{n} e^{i A t} f\right\|+\left\|\left(P-P_{n}\right) e^{i A t} f\right\| \\
\leq\left\|P_{n} e^{i \Delta t} f\right\|+\varepsilon\|f\| .
\end{gathered}
$$

But 


$$
\left\|P_{n} e^{i \Delta t} f\right\|^{2}=\sum_{j=1}^{n} \lambda_{j}^{2}\left|\left(e^{i \Delta t} f, \phi_{j}\right)\right|^{2},
$$

which, by 4.2 (ii), goes to 0 as $t \rightarrow \infty$. Since $\varepsilon$ is arbitrary, the proof is complete.

LEMMA 5.3. For all real $s, e^{-i B s} U=U e^{-i A s}$.

Proof.

$$
\begin{aligned}
e^{-i B s} U_{t}-U_{t} e^{-i A s} & =e^{-i B s} e^{-i B t} e^{i A t}-e^{-i B t} e^{i A t} e^{-i A s} \\
& ==e^{-i B t}\left(e^{-i B s}-e^{-i A s}\right) e^{i A t},
\end{aligned}
$$

which by 3.3 (ii)

$$
=\frac{1}{i} \int_{0}^{s} e^{-i B(x+t)} P e^{i A(x+t-s)} d x .
$$

Thus

$$
\begin{aligned}
& \left|\left(e^{-i B s} U_{t} f, g\right)-\left(U_{t} e^{-i A s} f, g\right)\right| \\
& \quad \leqq \int_{0}^{s}\left|\left(e^{-i B(x+t)} P e^{i A(x+t-s)} f, g\right)\right| d x \\
& \leqq \int_{0}^{s}\left\|P e^{i A(x+t-s)} f\right\| \cdot\|g\| d x .
\end{aligned}
$$

By the preceding lemma and the bounded convergence theorem this last term goes to 0 as $t \rightarrow \infty$. Since $U_{t}$ converges weakly to $U$ we have $\left(e^{-i B s} U f, g\right)-\left(U e^{-i A s} f, g\right)=0$, or 5.3 .

THEOREM 5.4. $B U=U A$.

Proof. By 5.3, $\left(e^{-i B s} U f, g\right)=\left(U e^{-t A s} f, g\right)$, or

$$
\int_{-\infty}^{\infty} e^{-i x s} d\left(F_{x} U f, g\right) / d x d x=\int_{-a}^{\infty} e^{-i x s} d\left(U E_{x:} f, g\right) / d x d x
$$

By the Fourier integral uniqueness theorem, $\left(F_{x} U f, g\right)=\left(U E_{x} f, g\right)$, and thus $B U=U A$.

\section{Conclusion of the proof.}

6.1. We know that as $t \rightarrow \infty, U_{t}$ converges weakly to $U$. Since $K_{n}(B) U_{t}$ converges strongly (Theorem 4.11) it follows that it converges strongly to $K_{n}(B) U$. From this we deduce that for all $f$ in $H$, 


$$
\lim _{t \rightarrow \infty}\left\|K_{n}(B) U_{t} f\right\|=\left\|K_{n}(B) U f\right\|
$$

and by 4.10 (iv),

(i) $\lim _{n \rightarrow \infty} \lim _{t \rightarrow \infty}\left\|K_{n}(B) U_{t} f\right\|=\|U f\|$.

We shall use 3.5 (i) in the form

(ii) $\left\|K_{n}(B) U_{t} f\right\|^{2}-\|f\|^{2}=\left(\left[K_{n}(B)-I\right] f, f\right)$

$$
\begin{aligned}
& +\frac{1}{i} \int_{0}^{t}\left(e^{-i \Delta x}\left[K_{n}(B)-I\right] P e^{i A x} f, f\right) d x \\
& -\frac{1}{i} \int_{0}^{t}\left(e^{-i \Delta x} P\left[K_{n}(B)-I\right] e^{i \Delta x} f, f\right) d x,
\end{aligned}
$$

to prove that

(iii) $\lim _{n \rightarrow \infty} \lim _{t \rightarrow \infty}\left\|K_{n}(B) U_{t} f\right\|=\|f\|$,

and thus show that $\|U f\|=\|f\|$. When this has been done we will have proved 2.1, and Theorem 1.6 will follow from the argument after 2.1. It is clear that it is sufficient to show that (iii) is valid for all $f$ is a dense set.

Definition 6.2. Let $D$ be the set of all $f$ in $H$ such that $d\left(E_{x} f, f\right) / d x$ is essentially bounded for all real $x$.

\section{THEOREM 6.3. $D$ is dense in $H$.}

Proof. Let $f$ be an arbitrary element in $H$. By 4.1 (i) $d\left(E_{x} f, f\right) / d x$ is almost everywhere finite. Let $M_{n}(x)$ be the characteristic function of the set of all real numbers $x$ such that $d\left(E_{x} f, f\right) / d x \leqq n$ or $d\left(E_{x} f, f\right) / d x$ $=\infty$. Then $M_{n}(A) f$ is a sequence of elements of $D$ that converges strongly to $f$. Hence 6.3 is true.

THEOREM 6.4. If $f \in D$, then 6.1 (iii) is true.

Proof. We shall consider each of the terms on the right hand side of 6.1 (ii). By 4.10 (iii), $\lim _{n \rightarrow \infty}\left(\left[K_{n}(B)-I\right] f, f\right)=0$.

By 4.4 (ii)

$$
\begin{aligned}
& \left|\begin{array}{l}
1 \\
i
\end{array} \int_{0}^{\infty}\left(e^{-i A x}\left[K_{n}(B)-I\right] P e^{i A x} f, f\right) d x\right| \\
& \leqq 2 \pi \operatorname{ess} \sup \frac{d\left(E_{x} f, f\right)}{d x}\left[\sum_{j=1}^{\infty}\left|\lambda_{j}\right|\left\|\left[K_{n}(B)-I\right] \phi_{j}\right\|^{2}\right]^{\frac{1}{2}}\left[\sum_{j=1}^{\infty}\left|\lambda_{j}\right|\right]^{\frac{1}{2}},
\end{aligned}
$$


which by 4.10 (iv) goes to 0 as $n \rightarrow \infty$. Thus it can be shown that all the terms on the right hand side of 6.1 (ii) go to 0 as $n \rightarrow \infty$, and thus 6.1 is true, and our proof of Theorem 1.6 is complete.

We conclude this paper with an interesting representation theorem for $F(B)-F(A)$.

Theorem 6.5. Assume 1.3-1.5 hold and that $F(x)$ is an essentially bounded function. Then

(i) $\lim _{t \rightarrow \infty}\left(e^{-i A t} F(B) e^{i A t} f, g\right)=(F(A) f, g)$ and

(ii) $\quad([F(B)-F(A)] f, g)=\lim _{t \rightarrow \infty} i \int_{0}^{t}\left(e^{-i \Delta x}[F(B) P-P F(B)] e^{i A x} f, g\right) d x$.

Proof. $\quad\left(e^{-i A t} F(B) e^{i A t} f, g\right)=\left(U_{t}^{*} F(B) U_{t} f, g\right)=\left(F(B) U_{t} f, U_{t} g\right)$. Since $U_{t} f$ and $U_{t} g$ converge strongly to $U f$ and $U g$ respectively and $U$ is unitary we have

$$
\lim _{t \rightarrow \infty}\left(e^{-i A t} F(B) e^{i A t} f, g\right)=(F(B) U f, U g)=(U F(A) f, U g)=(F(A) f, g) .
$$

(ii) is a consequence of (i) and Theorem 3.4.

\section{REFERENCES}

1. K. O. Friedrichs, Über die Speltralzerlegung eines Integraloperators, Math. Ann., 115 (1938), 249-272.

2. - On the perturbation of the continuous spectra, Communications on Applied Math., 1 (1948), 361-401.

3. E. Hille, Functional analysis and semi-groups, New York, 1948.

4. I. Kay and H. E. Moss, The determination of the scattering potential from the spectral measure function, N. Y. U. Research Report CX-18; January, 1955.

5. H. E. Moses, The scattering operator and the adiabatic theorem, Nuovo Cimento, 1 (1955), 103-131.

6. Riesz, F. and B. Sz.-Nagy, Lecons d'analyse fonctionnelle, Budapest, 1952.

7. M. H. Stone, Linear transformations in Hilbert space, New York, 1932.

8. J. von Neumann, Charakterisierung des Spektrums eines Integraloperators, Actualités

Sci. Ind., 229, 1935.

UNIVERSITY OF CALIFORNIA AND UNIVERSITY OF VIRGINIA 


\section{PACIFIC JOURNAL OF MATHEMATICS}

EDITORS

H. L. ROYDEN

Stanford University

Stanford, California

R. A. Beaumont

University of Washington

Seattle 5 , Washington
A. R. Whiteman

University of Southern California

Los Angeles 7, California

E. G. Straus

University of California

Los Angeles 24, California

\section{ASSOCIATE EDITORS}
E. F. BECKENBACH
C. E. BURGESS
M. HALL
E. HEWITT
A. HORN
V. GANAPATHY IYER
R. D. JAMES
M. S. KNEBELMAN

L. NACHBIN

I. NIVEN

G. SZEKERES

T. G. OSTROM

M. M. SCHIFFER
F. WOLF

K. YOSIDA

\section{SUPPORTING INSTITUTIONS}

UNIVERSITY OF BRITISH COLUMBIA

CALIFORNIA INSTITUTE OF TECHNOLOGY

UNIVERSITY OF CALIFORNIA

MONTANA STATE UNIVERSITY

UNIVERSITY OF NEVADA

OREGON STATE COLLEGE

UNIVERSITY OF OREGON

UNIVERSITY OF SOUTHERN CALIFORNIA

\author{
STANFORD UNIVERSITY \\ UNIVERSITY OF UTAH \\ WASHINGTON STATE COLLEGE \\ UNIVERSITY OF WASHINGTON \\ AMERICAN MATHEMATICAL SOCIETY \\ CALIFORNIA RESEARCH CORPORATION \\ HUGHES AIRCRAFT COMPANY \\ THE RAMO-WOOLDRIDGE CORPORATION
}




\section{Pacific Journal of Mathematics}

\section{Vol. 7, No. $1 \quad$ January, 1957}

Richard Davis Anderson, Zero-dimensional compact groups of

homeomorphisms ................................... 797

Hans-Joachim Bremermann, Holomorphic functionals and complex

convexity in Banach spaces........................... 811

Hugh D. Brunk, G. M. Ewing and W. R. Utz, Minimizing integrals in

certain classes of monotone functions ................. 833

Philip David, Uniqueness theory for asymptotic expansions in general

regions ...................................... 849

Paul Erdős and Harold Nathaniel Shapiro, On the least primitive root of a

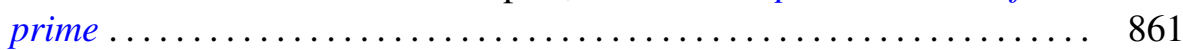

Watson Bryan Fulks, Regular regions for the heat equation ........... 867

William Robert Gaffey, A real inversion formula for a class of bilateral

Laplace transforms ................................ 879

Ronald Kay Getoor, On characteristic functions of Banach space valued random variables ................................. 885

Louis Guttman, Some inequalities between latent roots and minimax (maximin) elements of real matrices ...................... 897

Frank Harary, The number of dissimilar supergraphs of a linear graph .... 903

Edwin Hewitt and Herbert S. Zuckerman, Structure theory for a class of convolution algebras .................................. 913

Amnon Jakimovski, Some Tauberian theorems . . . . . . . . . . . . . . . . . 943

C. T. Rajagopal, Simplified proofs of "Some Tauberian theorems" of Jakimovski................................

Paul Joseph Kelly, A congruence theorem for trees ................. 961

Robert Forbes McNaughton, Jr., On the measure of normal formulas...... 969

Richard Scott Pierce, Distributivity in Boolean algebras .............. 983

Calvin R. Putnam, Continuous spectra and unitary equivalence ......... 993

Marvin Rosenblum, Perturbation of the continuous spectrum and unitary

equivalence................................... 997

V. N. Singh, Certain generalized hypergeometric identities of the

Rogers-Ramanujan type.......................

Peter Swerling, Families of transformations in the function spaces $H^{p} \ldots \ldots 1015$ 